\title{
Some ergodic problems for differential games
}

\author{
Martino Bardi* \\ Università di Padova \\ E-mail: bardi@math.unipd.it
}

\section{Olivier Alvarez}

Université de Rouen, France

E-mail: Olivier.alvarez@univ-rouen.fr

\begin{abstract}
We present a notion of ergodicity for deterministic zero-sum differential games that extends the one in classical ergodic control theory to systems with two conflicting controllers. We describe its connections with the existence of a constant and uniform long-time limit of the value function of finite horizon games, and characterize this property in terms of Hamilton-Jacobi-Isaacs equations. We also give several sufficient conditions for ergodicity and describe some extensions of the theory to stochastic differential games.
\end{abstract}




\section{Introduction}

We consider a nonlinear system in $\mathbb{R}^{m}$ controlled by two players

$$
\dot{y}(t)=f(y(t), a(t), b(t)), \quad y(0)=x,
$$

and we denote with $y_{x}(\cdot)$ the trajectory starting at $x$. We are also given a bounded, uniformly continuous running cost $l$, and we are interested in the payoffs associated to the long time average cost (briefly, LTAC), namely,

$$
\begin{aligned}
J^{\infty}(x, a(\cdot), b(\cdot)) & :=\limsup _{T \rightarrow \infty} \frac{1}{T} \int_{0}^{T} l\left(y_{x}(t), a(t), b(t)\right) d t, \\
J_{\infty}(x, a(\cdot), b(\cdot)) & :=\liminf _{T \rightarrow \infty} \frac{1}{T} \int_{0}^{T} l\left(y_{x}(t), a(t), b(t)\right) d t .
\end{aligned}
$$

We denote with $u-\operatorname{val} J^{\infty}(x)$ (respectively, $l-\operatorname{val} J_{\infty}(x)$ ) the upper value of the zero-sum game with payoff $J^{\infty}$ (respectively, the lower value of the game with payoff $J_{\infty}$ ) which the 1st player $a(\cdot)$ wants to minimize while the 2 nd player $b(\cdot)$ wants to maximize, and the values are in the sense of Variya-Roxin-Elliott-Kalton. We say that the LTAC game is ergodic if

$$
u-\operatorname{val} J^{\infty}(x)=l-\operatorname{val} J_{\infty}(x)=\lambda \quad \forall x,
$$

for some constant $\lambda$.

The terminology is motivated by the analogy with classical ergodic control theory, see, e.g., [11, 26, 14, 10, 22, 7, 8, and by the fact that for uncontrolled system and cost the game is ergodic for all continuous $l$ if the dynamical system $\dot{y}=f(y)$ is ergodic with a unique invariant measure (see Proposition 13 of [3] for a precise statement). Similar problems were already studied for some games, in particular by Fleming and McEneaney [19] in the context of risk-sensitive control, Carlson and Haurie [13] within the turnpike theory, and Kushner [27] for controlled nondegenerate diffusion processes. There is a large literature on related problems for dicrete-time games, see the recent survey by Sorin [33.

In order to have a compact state space we assume that the data $f$ and $l$ are $\mathbb{Z}^{m}$-periodic. First of all we show the connection between the ergodicity of the LTAC game and the existence of a constant and uniform long-time limit of the lower and upper value functions of the fi nite horizon games with the same running cost. We call this property ergodicity of the lower (respectively, upper) game. Then we prove that the lower game is ergodic with limit $\lambda$ if and only if the lower value of the discounted infi nite horizon game with payoff

$$
\delta \int_{0}^{\infty} l\left(y_{x}(t), a(t), b(t)\right) e^{-\delta t} d t
$$

converges uniformly to $\lambda$ as the discount rate $\delta$ tends to 0 . Moreover, this is also equivalent to the existence of a $\mathbb{Z}^{m}$-periodic viscosity $\chi$ to the Hamilton-Jacobi-Isaacs equation

$$
\lambda+\min _{b} \max _{a}\{-f(y, a, b) \cdot \nabla \chi-l(y, a, b)\}=0,
$$

and similar statements hold for the upper value. 
Next we describe two sets of conditions ensuring the previous facts and therefore the ergodicity. The fi rst is a bounded-time controllability property of the system by one of the players, uniformly with respect to the behavior of the opponent. It is a generalization to games of a condition used for systems with a single controller by Grüne [22], Arisawa [7], and Artstein and Gaitsgory [9].

Different from the first, the second set of conditions is symmetric for the two players. We assume that some state variables $y_{A}$ are asymptotically controllable by the first player, and the remaining variables $y_{B}$ are asymptotically controllable by the second (see Section 2 for the precise defi nition). In this case neither player can control the whole state vector $y=\left(y_{A}, y_{B}\right)$. We further assume the running cost depends only on $y^{A}$ and $y^{B}$ and has a saddle point, namely,

$$
\min _{y^{A}} \max _{y^{B}} l\left(y^{A}, y^{B}\right)=\max _{y^{B}} \min _{y^{A}} l\left(y^{A}, y^{B}\right)=: \bar{l},
$$

Then we show that the LTAC game has the value $\lambda=\bar{l}$.

In the last section we also show that for systems affected by a non-degenerate white noise the game is ergodic with no controllability assumptions on either player (see [27] for related results).

Our methods rely heavily on the Hamilton-Jacobi-Isaacs equations associated to the games, in the framework of the theory of viscosity solutions. We follow ideas of authors such as P.-L. Lions and L.C. Evans, see [28, 16, 10, 8], and their developments in our papers [3, 4].

Undiscounted infi nite horizon control problems arise in many applications to economics and engineering, see [14, 11, 26] and [13, 19, 33] for games. Our additional motivation is that ergodicity plays a crucial role in the theory of singular perturbation problems for the dimension reduction of multiple-scale systems [25, 11, 26, 24, 21, 34, 35, 30] and for the homogenization in oscillating media [28, 16, 17, 1, 23, 29, 12, 6. A general principle emerging in the papers

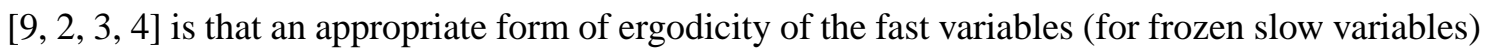
ensures the convergence of the singular perturbation problem, in a suitable sense.

The paper is organized as follows. Section 1 describes the connection between the ergodicity of the LTAC game and the ergodicity of the lower and upper game. Section 2 studies the ergodicity of the fi nite horizon games. Section 3 presents some examples. In Section 4 we give some extensions of the results of Section 2 to diffusion processes controlled by two players, and we prove the ergodicity result for nondegenerate noise.

The full proofs of the results of this paper can be found in [5].

\section{The long-time-average-cost game and ergodicity}

About the system (1) and the cost we assume throughout the paper that $f: \mathbb{R}^{m} \times A \times B \rightarrow \mathbb{R}^{m}$ and $l: \mathbb{R}^{m} \times A \times B \rightarrow \mathbb{R}$ are continuous and bounded, $A$ and $B$ are compact metric spaces, $f$ is lipschitzean in $x$ uniformly in $a, b$. In this section we do not assume the compactness of the state space.

We consider the cost funtional

$$
J(T, x)=J(T, x, a(\cdot), b(\cdot)):=\frac{1}{T} \int_{0}^{T} l\left(y_{x}(t), a(t), b(t)\right) d t,
$$

where $y_{x}(\cdot)$ is the trajectory corresponding to $a(\cdot)$ and $b(\cdot)$. We denote with $\mathcal{A}$ and $\mathcal{B}$, respectively, the sets of open-loop (measurable) controls for the first and the second player, and with $\Gamma$ and 
$\Delta$, respectively, the sets of nonanticipating strategies for the fi rst and the second player, see, e.g., [18, 10] for the precise defi nition. Finally, we defi ne the upper and lower values for the fi nite horizon game with average cost

$$
\begin{aligned}
& u-\operatorname{val} J(T, x):=\sup _{\beta \in \Delta} \inf _{a \in \mathcal{A}} J(T, x, a, \beta[a]), \\
& l-\operatorname{val} J(T, x):=\inf _{\alpha \in \Gamma} \sup _{b \in \mathcal{B}} J(T, x, \alpha[b], b),
\end{aligned}
$$

and for the LTAC game

$$
\begin{gathered}
u-\operatorname{val} J^{\infty}(x):=\sup _{\beta \in \Delta} \inf _{a \in \mathcal{A}} \limsup _{T \rightarrow \infty} J(T, x, a, \beta[a]), \\
l-\operatorname{val} J_{\infty}(x):=\inf _{\alpha \in \Gamma} \sup _{b \in \mathcal{B}} \liminf _{T \rightarrow \infty} J(T, x, \alpha[b], b) .
\end{gathered}
$$

We say that the the lower game is (uniformly) ergodic if the long time limit of the fi nite horizon value exists, uniformly in $x$, and it is constant, i.e.,

$$
l-\operatorname{val} J(T, \cdot) \rightarrow \lambda \quad \text { as } T \rightarrow \infty \text { uniformly in } \mathbb{R}^{m}
$$

Similarly, the upper game is ergodic if

$$
u-\operatorname{val} J(T, \cdot) \rightarrow \Lambda \quad \text { as } T \rightarrow \infty \text { uniformly in } \mathbb{R}^{m}
$$

Theorem 1 If the lower game is ergodic, then

$$
l-\operatorname{val} J_{\infty}(x)=\lim _{T \rightarrow \infty} l-\operatorname{val} J(T, x)=\lambda \quad \forall x \in \mathbb{R}^{m} ;
$$

if the upper game is ergodic, then

$$
u-\operatorname{val} J^{\infty}(x)=\lim _{T \rightarrow \infty} u-\operatorname{val} J(T, x)=\Lambda \quad \forall x \in \mathbb{R}^{m}
$$

We recall the classical Isaacs' condition, or solvability of the small game,

$H(y, p):=\min _{b \in B} \max _{a \in A}\{-f(y, a, b) \cdot p-l(y, a, b)\}=\max _{a \in A} \min _{b \in B}\{-f(y, a, b) \cdot p-l(y, a, b)\}, \quad \forall y, p \in \mathbb{R}^{m}$.

It is well known that it implies the equality of the upper and the lower value of the fi nite horizon game, that is, the existence of the value of that game, which we denote with val $J(T, x)$, see [18, 10]. Therefore we immediately get the following consequence of Theorem it.

Corollary 1 Assume (1.3) and that either the lower or the upper game is ergodic. Then the LTAC game is ergodic, i.e.,

$$
l-\operatorname{val} J_{\infty}(x)=u-\operatorname{val} J^{\infty}(x)=\lim _{T \rightarrow \infty} \operatorname{val} J(T, x)=\lambda, \quad \forall x \in \mathbb{R}^{m}
$$




\section{Characterizations of ergodicity}

From now on we add periodicity to the standing assumptions:

$$
f(y, a, b)=f(y+k, a, b), \quad l(y, a, b)=l(y+k, a, b), \quad \forall k \in \mathbb{Z}^{m}, y \in \mathbb{R}^{m}, a \in A, b \in B .
$$

This means that the state space is the $m$-torus $\mathbb{T}^{m}=\mathbb{R}^{m} / \mathbb{Z}^{m}$. The first result is a consequence of Theorem 4 in [3].

Theorem 2 The following statements on the lower game are equivalent.

(i) The lower game is ergodic, i.e., $l-\operatorname{val} J(T, x) \rightarrow$ const uniformly in $x$ as $T \rightarrow+\infty$.

(ii) $l-\operatorname{val} \delta \int_{0}^{\infty} l\left(y_{x}(t), a(t), b(t)\right) e^{-\delta t} d t \rightarrow$ const uniformly in $x$ as $\delta \rightarrow 0+$.

(iii) The additive eigenvalue problem

$$
\lambda+\min _{b \in B} \max _{a \in A}\{-f(y, a, b) \cdot \nabla \chi-l(y, a, b)\}=0 \quad \text { in } \mathbb{R}^{m}, \quad \chi \mathbb{Z}^{m} \text {-periodic }
$$

has the property that

$$
\begin{aligned}
\sup \{\lambda \mid \text { there is a viscosity subsolution of }(2.2)\} \\
\qquad=\inf \{\lambda \mid \text { there is a viscosity supersolution of (2.2) }\} .
\end{aligned}
$$

If one of the above assertions is true, then the constants in (i) and (ii) are equal and they coincide with the number defined by (2.3). Moreover, the same result holds for the upper game, after replacing $l$ - val with $u$ - val in (i) and (ii), and (2.2) with

$$
\lambda+\max _{a \in A} \min _{b \in B}\{-f(y, a, b) \cdot \nabla \chi-l(y, a, b)\}=0 \quad \text { in } \mathbb{R}^{m}, \quad \chi \mathbb{Z}^{m} \text {-periodic. }
$$

Proof It is well known [18, 10] that $w(t, y):=t(l-\operatorname{val} J(t, y))$ is the viscosity solution of the Cauchy problem for the Isaacs equation

$$
w_{t}+H\left(y, D_{y} w\right)=0 \quad \text { in }(0,+\infty) \times \mathbb{R}^{m}, \quad w(0, y)=0, \quad w \text { periodic } .
$$

The equivalence of (iii) and the uniform convergence of $w(t, \cdot) / t$ to a constant as $t \rightarrow \infty$ is stated in Theorem 4 of [3], and it gives the equivalence of (i) and (iii).

Next, $w_{\delta}(x):=l-\operatorname{val} \int_{0}^{\infty} l\left(y_{x}(t), a(t), b(t)\right) e^{-\delta t} d t$ is the viscosity solution of the Isaacs equation

$$
\delta w_{\delta}+H\left(y, D w_{\delta}\right)=0 \quad \text { in } \mathbb{R}^{m}, \quad w_{\delta} \text { periodic },
$$

and Theorem 4 of [⿰] $]$ states the equivalence of (iii) and the uniform convergence of $\delta w_{\delta}$ to a constant as $\delta \rightarrow 0+$. Therefore (ii) and (iii) are equivalent.

The equality of the three constants is also given by Theorem 4 of [3]. Finally, the proof for the upper value is the same, with the Hamiltonian $H=\min \max$ replaced by max min. 
REMARK Note that (ii) deals with a vanishing discount rate problem for infi nite horizon games. The equivalence between (i) and (ii) is a differential game extension of the classical AbelianTauberian theorem, stating that

$$
\lim _{T \rightarrow \infty} \frac{1}{T} \int_{0}^{T} \varphi(t) d t=\lim _{\delta \rightarrow 0+} \delta \int_{0}^{\infty} \varphi(t) e^{-\delta t} d t
$$

whenever one of the two limits exists. The property (iii) is a characterization of the uniform ergodicity of the lower game by a Hamilton-Jacobi-Isaacs equation. In some cases the inf and the sup in the formula (2.3) are attained and the number defi ned by 2.3$)$ is the unique constant $\lambda$ such that the additive eigenvalue problem (2.2) has a continuous viscosity solution, see the remark in Section 3. In general, however, even if (iii) holds, (2.2) may have no continuous solution $\chi$ (see Arisawa, Lions [8]). By analogy with the theory of homogenization we call (2.2) the cell problem.

Whenever the conditions of Corollary 1 for the ergodicity of the LTAC game are satisfi ed, we have the following informations on the value of the game, namely, the constant $\lambda$.

Proposition 1 Assume (1.3) and that either the lower or the upper game is ergodic. Then $\lambda=$ $l-\operatorname{val} J_{\infty}(x)=u-\operatorname{val} J^{\infty}(x)$ satisfies

$$
\min _{x} \min _{a \in A} \max _{b \in B} l(x, a, b) \leq \lambda \leq \max _{x} \min _{a \in A} \max _{b \in B} l(x, a, b) .
$$

If, moreover,

$$
\max _{a \in A} \min _{b \in B}\{-f(x, a, b) \cdot p-l(x, a, b)\} \geq \max _{a \in A} \min _{b \in B}\{-l(x, a, b)\} \quad \forall x, p \in \mathbb{R}^{m},
$$

(respectively, $\leq$ ), then

$$
\lambda=\min _{x} \min _{a \in A} \max _{b \in B} l(x, a, b),
$$

(respectively, $\lambda=\max _{x} \min _{a} \max _{b} l(x, a, b)$ ).

ProOF First we use the characterization (i) of ergodicity in Theorem 2, and we set $w(t, y):=$ $t \cdot l-\operatorname{val} J(t, y)$. It is well known [18, 10] that $w$ satisfi es, in the viscosity sense,

$$
w_{t}+H\left(y, D_{y} w\right)=0 \quad \text { in }(0,+\infty) \times \mathbb{R}^{m}, \quad w(0, y)=0, \quad w \text { periodic } .
$$

We observe that $-t \max _{y} H(y, 0)$ and $-t \min _{y} H(y, 0)$ are, respectively, a sub- and a supersolution of this Cauchy problem. Therefore the comparison principle gives

$$
-t \max _{y} H(y, 0) \leq w(t, y) \leq-t \min _{y} H(y, 0)
$$

We divide by $t$ and let $t \rightarrow+\infty$. Since $w(t, y) / t \rightarrow \lambda$, and $H(y, 0)=\max _{a} \min _{b}\{-l(x, a, b)\}$ by (1.3), we get the first pair of inequalities.

To prove the second statement we assume by contradiction that $\lambda>-H(y, 0)$ in a neighborhood of a minimum point of $-H(y, 0)=\min _{a} \max _{b} l(x, a, b)$. Now we use the characterization (ii) of ergodicity in Theorem 2 as in the proof of Theorem 3 . With the same notations, the value 
function $w_{\delta}$ of the infi nite horizon discounted game satisfi es the Isaacs equation (2.12). By the uniform convergence of $\delta w_{\delta}$ to $\lambda$ we get

$$
H\left(y, D w_{\delta}\right)-H(y, 0)=-\lambda-H(y, 0)+o(1)<0 \quad \text { as } \delta \rightarrow 0
$$

in an open set. This is a contradiction with the assumption (2.4).

REMARK Note that, for a running cost independent of the controls, $l=l(x)$, the condition (2.4) reads

$$
\min _{a} \max _{b} f(x, a, b) \cdot p=\max _{b} \min _{a} f(x, a, b) \cdot p \leq 0,
$$

in view of (1.3). This says that the fi rst player has a stronger control on the vector fi eld than the second. The conclusion is that the LTAC value is

$$
\lambda=\min _{x} l(x),
$$

so the minimizing player can drive asymptotically the system near the minimum points of the running cost.

Next we describe some suffi cient conditions for the ergodicity of the upper or the lower game. We say that the system (11) is bounded-time controllable by the first player if for some $S>0$ and for all $x, \widetilde{x} \in \mathbb{R}^{m}$ there is a strategy $\widetilde{\alpha} \in \Gamma$ such that for all control functions $b \in \mathcal{B}$

$$
\exists t^{\#}=t^{\#}(x, \widetilde{x}, \widetilde{\alpha}, b) \leq S \text { such that } y_{x}\left(t^{\#}\right)-\widetilde{x} \in \mathbb{Z}^{m},
$$

where $y_{x}(\cdot)$ is the trajectory corresponding to the strategy $\widetilde{\alpha}$ and the control function $b$, i.e., it solves

$$
\dot{y}(t)=f(y(t), \widetilde{\alpha}[b](t), b(t)), \quad y(0)=x .
$$

In other words, the first player can drive the system from any initial position $x$ to any given state $\tilde{x}$ on the torus $\mathbb{T}^{m}$ in a uniformly bounded time for all possible behaviors of the second player. Symmetrically, we say that he system (1) is bounded-time controllable by the second player if for some $S>0$ and for all $x, \widetilde{x} \in \mathbb{R}^{m}$ there is a strategy $\widetilde{\beta} \in \Delta$ such that for all control functions $a \in \mathcal{A}$

$$
\exists t^{\#}=t^{\#}(x, \widetilde{x}, a, \widetilde{\beta}) \leq S \text { such that } y_{x}\left(t^{\#}\right)-\widetilde{x} \in \mathbb{Z}^{m},
$$

where $y_{x}(\cdot)$ is the trajectory corresponding to the strategy $\widetilde{\beta}$ and the control function $a$, i.e., it solves

$$
\dot{y}(t)=f(y(t), a(t), \widetilde{\beta}[a](t)), \quad y(0)=x .
$$

For systems with a single player this notion is studied in the literature under various names such as complete controllability [15], uniform exact controllability [7], and total controllability [9].

Theorem 3 If the system (1) is bounded-time controllable by the first player (respectively, by the second player), then the lower game (respectively, the upper game) is ergodic. 
PROOF The proof uses the characterization (ii) of ergodicity in Theorem 2 and we call $w_{\delta}$ the lower value function of the discounted infi nite horizon problem, namely,

$$
w_{\delta}(x):=\inf _{\alpha \in \Gamma} \sup _{b \in \mathcal{B}} \int_{0}^{\infty} l\left(y_{x}(t), \alpha[b](t), b(t)\right) e^{-\delta t} d t .
$$

The main tool of the proof is the following Dynamic Programming Principle due to Soravia, Remark 4.2 of [32],

$$
w_{\delta}(x)=\inf _{\alpha \in \Gamma} \sup _{b \in \mathcal{B}} \inf _{0 \leq t<\infty}\left\{\int_{0}^{t} l(y(s), \alpha[b](s), b(s)) e^{-\delta s} d s+e^{-\delta t} w_{\delta}(y(t))\right\},
$$

where $y(\cdot)$ is the trajectory of (2.8) with $\widetilde{\alpha}$ replaced by a generic $\alpha$.

For fi xed $x \widetilde{x}$ we take a strategy $\widetilde{\alpha} \in \Gamma$ such that (2.7) holds. Then (2.9) and the periodicity of $w_{\delta}$ give

$$
w_{\delta}(x) \leq \sup _{b \in \mathcal{B}}\left\{\int_{0}^{t^{\#}} l(y(s), \alpha[b](s), b(s)) e^{-\delta s} d s+e^{-\delta t^{\#}} w_{\delta}(\widetilde{x})\right\},
$$

where $y(\cdot)$ is the trajectory of (2.8). Since $l$ and $\delta w_{\delta}$ are uniformly bounded, there is a constant $C$ such that

$$
\delta w_{\delta}(x)-\delta w_{\delta}(\widetilde{x}) \leq C\left(1-e^{-\delta S}\right) .
$$

Now we exchange the roles of $x$ and $\tilde{x}$ to get

$$
\lim _{\delta \rightarrow 0+}\left|\delta w_{\delta}(x)-\delta w_{\delta}(\widetilde{x})\right|=0 \quad \text { uniformly in } x, \widetilde{x} \in \mathbb{R}^{m} .
$$

If for fi xed $\widetilde{x}$ we choose a sequence $\delta_{k} \rightarrow 0$ such that $\delta_{k} w_{\delta_{k}}(\widetilde{x}) \rightarrow \mu$, we obtain the uniform convergence of $\delta_{k} w_{\delta_{k}}$ to $\mu$.

We claim that $\mu$ is independent of the sequence $\delta_{k}$. This implies the uniform convergence of the whole net $\delta w_{\delta}$ to $\mu$, as desired. To prove the claim we recall the cell problem (2.2), i.e.,

$$
\lambda+H(y, D \chi)=0 \quad \text { in } \mathbb{R}^{m}, \quad \chi \text { periodic, }
$$

where $\lambda$ is a constant, and use the inequality

$\lambda_{1}:=\sup \{\lambda \mid \exists$ a u.s.c. subsolution of $(2.11)\} \leq \lambda_{2}:=\inf \{\lambda \mid \exists$ a l.s.c. supersolution of (2.11) $\}$,

which follows from a standard argument based on the comparison principle for sub- and supersolutions of Hamilton-Jacobi equations (see, e.g., the proof of Theorem 1 in [7] or that of Theorem 4 in [3]). The Isaacs equation satisfi ed by $w_{\delta}$ in viscosity sense is (see, e.g., [10])

$$
\delta w_{\delta}+H\left(y, D w_{\delta}\right)=0 \quad \text { in } \mathbb{R}^{m}, \quad w_{\delta} \text { periodic. }
$$

Then, for $\lambda<\mu, w_{\delta_{k}}$ is a subsolution of (2.11) for $k$ large enough, so $\mu \leq \lambda_{1}$. The same argument gives $\lambda_{2} \leq \mu$. Therefore $\mu=\lambda_{1}=\lambda_{2}$, which proves the claim.

An immediate consequence of this theorem and of Corollary 1 is the following.

Corollary 2 Assume the Isaacs' condition (1.3) and that the system (11) is bounded-time controllable either by the first or by the second player. Then the LTAC game is ergodic, i.e.,

$$
l-\operatorname{val} J_{\infty}(x)=u-\operatorname{val} J^{\infty}(x)=\lim _{T \rightarrow \infty} \operatorname{val} J(T, x)=\lambda, \quad \forall x \in \mathbb{R}^{m} .
$$


We end this section with some suffi cient conditions for ergodicity that are symmetric for the two players, different from the preceding Theorem 3 and Proposition 11 where one of the two players have a much stronger hold of the system than the other. We take a system of the form

$$
\begin{cases}\dot{y}^{A}(t)=f_{A}(y(t), a(t), b(t)), & y^{A}(0)=x^{A} \in \mathbb{R}^{m_{A}}, \\ \dot{y}^{B}(t)=f_{B}(y(t), a(t), b(t)), & y^{B}(0)=x^{B} \in \mathbb{R}^{m_{B}}, \\ y(t)=\left(y^{A}(t), y^{B}(t)\right), & \end{cases}
$$

and we assume that the state variables $y^{A}$ are (uniformly) asymptotically controllable by the first player, whereas the variables $y^{B}$ are asymptotically controllable by the second, in the following sense. There exists a function $\eta:[0, \infty) \rightarrow[0, \infty)$ with

$$
\lim _{T \rightarrow \infty} \eta(T)=0,
$$

and for all $x^{A}, \widetilde{x}^{A} \in \mathbb{R}^{m_{A}}, x^{B} \in \mathbb{R}^{m_{B}}$, there is a strategy $\widetilde{\alpha} \in \Gamma$ such that, for $x=\left(x^{A}, x^{B}\right)$,

$$
\frac{1}{T} \int_{0}^{T} \min _{k^{A} \in \mathbb{Z}^{m_{A}}}\left|y_{x}^{A}(t)-\widetilde{x}^{A}-k^{A}\right| d t \leq \eta(T), \forall b \in \mathcal{B},
$$

whereas for all $x^{B}, \widetilde{x}^{B} \in \mathbb{R}^{m_{B}}, x^{A} \in \mathbb{R}^{m_{A}}$, there is a strategy $\widetilde{\beta} \in \Delta$ such that

$$
\frac{1}{T} \int_{0}^{T} \min _{k^{B} \in \mathbb{Z}^{m_{B}}}\left|y_{x}^{B}(t)-\widetilde{x}^{B}-k^{B}\right| d t \leq \eta(T), \forall a \in \mathcal{A} .
$$

Note that the integrand in (2.15) is the distance between $y_{x}^{A}(t)$ and $\widetilde{x}^{A}$ on the $m_{A}$-dimensional torus $\mathbb{T}^{m_{A}}=\mathbb{R}^{m_{A}} / \mathbb{Z}^{m_{A}}$, so (2.15) and (2.14) mean that the fi rst player can drive asymptotically $y^{A}$ near $\widetilde{x}^{A}$, uniformly with respect to $x, \widetilde{x}^{A}$, and the control of the other player $b$. Similarly, (2.16) says that the second player can drive asymptotically $y^{B}$ to $\widetilde{x}^{B}$ on the $m_{B}$-dimensional torus $\mathbb{T}^{m_{B}}$, uniformly with respect to $x, \widetilde{x}^{B}$, and $a$.

We will also assume that the running cost does not depend on the controls, $l=l\left(y^{A}, y^{B}\right)$, and it has a saddle point in $[0,1]^{m_{A}} \times[0,1]^{m_{B}}$, that is, it satisfi es

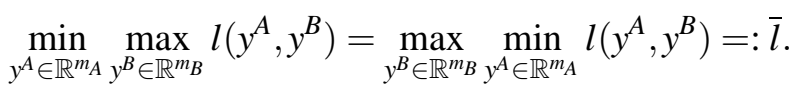

Proposition 2 Assume the system (1) is of the form (2.13) with $y^{A}$ and $y^{B}$ asymptoticallly controllable, respectively, by the first and by the second player. Suppose also that $l=l\left(y^{A}, y^{B}\right)$ satisfies (2.17) and (1.3) holds. Then the LTAC game is ergodic and its value is the value of the static game with payoff $l$, that is,

$$
l-\operatorname{val} J_{\infty}\left(x^{A}, x^{B}\right)=u-\operatorname{val} J^{\infty}\left(x^{A}, x^{B}\right)=\bar{l}, \quad \forall\left(x^{A}, x^{B}\right) \in \mathbb{R}^{m} .
$$

REMARK If the system governing $y^{A}$ is bounded-time controllable by the fir rst player and also stoppable, i.e.,

$$
\forall x \in \mathbb{R}^{m}, \forall b \in B, \exists a \in A: f_{A}(x, a, b)=0,
$$

then the variables $y^{A}$ are asymptotically controllable, because $\widetilde{x}^{A}$ can be reached from $x^{A}$ in a time smaller than $S$ and then the first player can keep $y^{A}(t)=\widetilde{x}^{A}$ for all later times $t$. In this case, if $\bar{l}=l\left(\widetilde{x}^{A}, \widetilde{x}^{B}\right)$, an optimal strategy for the fi rst player amounts to driving the variables $y^{A}$ to the saddle point $\widetilde{x}^{A}$ and stopping there, and the strategy of going to $\widetilde{x}^{B}$ and staying there forever is optimal for the second player. This kind of behavior is called a turnpike, see [14, 13. 


\section{Examples}

Example 1: first order controllability. Assume that for some $v>0$

$$
B(0, v ; m) \subset \overline{\operatorname{conv}}\{f(x, a, b) \mid a \in A\}, \quad \forall x \in \mathbb{R}^{m}, b \in B,
$$

where $B(0, v: m)$ denotes the $m$-dimensional open ball of radius $v$ centered at the origin and $\overline{c o n v}$ the closed convex hull. From the standard theory of differential games (see, for instance, Corollary 3.7 in [31]) it is known that the system is (small-time) controllable by the fi rst player and the time necessary to reach a point $\widetilde{x}$ from $x$ satisfi es an estimate of the form

$$
t^{\#}(x, \tilde{x}, \widetilde{\alpha}, b) \leq \frac{C}{v}|x-\widetilde{x}| .
$$

Therefore the lower game is uniformly ergodic in this case. Moreover, if $l=l(x)$ it is easy to see that (2.6) holds, so $\lambda=\min _{x} l(x)$.

Example 2: higher order controllability. Consider a system of the form

$$
\dot{y}(t)=\sum_{i=1}^{k-1} a_{i}(t) g^{i}(y(t))+a_{k}(t) g^{k}(y(t), a(t), b(t)),
$$

where the control of the fi rst player $a=\left(a_{1}, \ldots, a_{k}\right)$ varies in a neighborhood of the origin $A \subset \mathbb{R}^{k}$, and all $g^{i}$ with $i \leq k-1$ are $C^{\infty}$ vector fi eld in $\mathbb{R}^{m}$. Moreover, we suppose the full rank (Hörmander) condition on $g^{1}, \ldots, g^{k-1}$, that is,

$$
\left\{\begin{array}{l}
\text { the vector fi elds } g^{1}, \ldots, g^{k-1} \\
\text { and their commutators of any order } \\
\text { span } \mathbb{R}^{m} \text { at each point of } \mathbb{R}^{m} .
\end{array}\right.
$$

By choosing $a_{k} \equiv 0$ we obtain a symmetric system independent of the second player. Then the classical Chow's theorem of geometric control theory says that this system is small-time locally controllable at all points of the state space. Moreover, for any small $t>0$ the reachable set from $x$ in time $t$ is a neighborhood of $x$, and the same holds for the reachable set backward in time. From this, using the compactness of the torus $\mathbb{T}^{m}$, it is easy to see that the whole state space is an invariant control set in the terminology of [15]. Then the global bounded-time controllability follows from Lemma 3.2.21 in [15]. In conclusion, the full system (3.2) is bounded-time controllable by the fi rst player and therefore the lower game is uniformly ergodic. As in the previous example, if $\lambda$ is independent of the controls (2.6) holds and $\lambda=\min _{x} l(x)$.

REMARK If (2.7) holds with $t^{\#}(x, \widetilde{x}, \widetilde{\alpha}, b) \leq \omega(|x-\widetilde{x}|)$ for all $|x-\widetilde{x}| \leq \gamma$ and all $b \in \mathcal{B}$, for some modulus $\omega$ and $\gamma>0$, we say that (1) is also small-time controllable by the first player. For such systems there exists a continuous solution $\chi$ to the additive eigenvalue problem (2.2), by Proposition 9.2 in [ [ ]. The systems of the Examples 1 and 2 are indeed small-time controllable by the fi rst player.

Example 3: separate controllability. For a system of the form (2.13) we can assume that the subsystem for the variables $y^{A}$ either satisfi es

$$
B\left(0, v ; m_{A}\right) \subset \overline{\operatorname{conv}} f(x, A, b) \quad \forall x \in \mathbb{R}^{m}, b \in B,
$$


or it is of the form

$$
\dot{y}^{A}=\sum_{i=1}^{k_{A}-1} a_{i} g_{A}^{i}\left(y^{A}\right)+a_{k_{A}} g_{A}^{k_{A}}(y, a, b),
$$

where the control of the fi rst player $a=\left(a_{1}, \ldots, a_{k_{A}}\right)$ varies in a neighborhood of the origin $A \subset \mathbb{R}^{k_{A}}$, and the vector fi elds $g_{A}^{1}, \ldots, g_{A}^{k_{A}-1}$ are of class $C^{\infty}$ and satisfy the full rank condition in $\mathbb{R}^{m_{A}}$. Then the variables $y^{A}$ are asymptotically controllable because the fi rst player can drive them from $x^{A}$ to $\widetilde{x}^{A}$ in bounded time and then stop there by choosing the null control. Similarly, the variables $y^{A}$ are asymptotically controllable if either $f_{B}$ verifi es

$$
B\left(0, v ; m_{A}\right) \subset \overline{\operatorname{conv}} f(x, a, B) \quad \forall x \in \mathbb{R}^{m}, a \in A,
$$

or it is of the form

$$
\dot{y}^{B}=\sum_{i=1}^{k_{B}-1} b_{i} g_{B}^{i}\left(y^{B}\right)+b_{k_{B}} g_{B}^{k_{B}}(y, a, b),
$$

where the control of the second player $b=\left(b_{1}, \ldots, b_{k_{B}}\right)$ varies in a neighborhood of the origin $B \subset \mathbb{R}^{k_{B}}$, and the vector fi elds $g_{B}^{1}, \ldots, g_{B}^{k_{B}-1}$ are of class $C^{\infty}$ and satisfy the full rank condition in $\mathbb{R}^{m_{B}}$. Under these condition and with $l=l\left(y^{A}, y^{B}\right)$ satisfying (2.17) Proposition 2 implies the ergodicity of the LTAC game and the formula (2.18).

\section{Ergodicity of noisy systems}

In this section we study the ergodicity of the lower value for the following class of stochastic differential games. We consider the controlled diffusion process

$$
d y(t)=f(y(t), a(t), b(t)) d t+\sigma(y(t), a(t), b(t)) d W(t), \quad y(0)=x,
$$

where $W$ is an $r$-dimensonal Brownian motion, and $\sigma$ is a continuous map from $\mathbb{R}^{m} \times A \times B$ to the space of $m \times r$ matrices, lipschitzean in $x$ uniformly in $a, b$. The fi nite horizon cost functional is

$$
J(T, x)=J(T, x, a(\cdot), b(\cdot)):=E\left[\frac{1}{T} \int_{0}^{T} l\left(y_{x}(t), a(t), b(t)\right) d t\right],
$$

where $E$ denotes the expectation. The set of admissible controls for the second player, that we still denote with $\mathcal{B}$, contains the progressively measurable functions of time taking values in $B$, and $\mathcal{A}$ will denote the admissible controls of the fi rst player. We also keep the notation $\Gamma$ and $\Delta$ for the set of nonanticipating admissible strategies for the fi rst player and the second player, respectively, and we refer to [20] for the precise defi nitions in the stochastic setting. The lower value of the fi nite horizon game is

$$
v(T, x):=l-\operatorname{val} J(T, x):=\inf _{\alpha \in \Gamma} \sup _{b \in \mathcal{B}} J(T, x, \alpha[b], b)
$$

and we say that the lower game is ergodic if

$$
l-\operatorname{val} J(T, \cdot) \rightarrow \lambda \quad \text { as } T \rightarrow \infty \text { uniformly in } \mathbb{R}^{m} .
$$

Besides the periodicity in the state variable of $f$ and $l$ (2.1) we assume

$$
\sigma(y, a, b)=\sigma(y+k, a, b), \quad \forall k \in \mathbb{Z}^{m}, y \in \mathbb{R}^{m}, a \in A, b \in B .
$$


We are going to extend all the results of Section 2 to this setting, and we also present a theorem where the ergodicity is due to the effects of the diffusion without any controllability hypothesis. Analogous results hold for the upper game, which is defi ned in the obvious way, but we will not state them explicitly.

We begin with the stochastic counterpart of the Abelian-Tauberian-type Theorem 2 that is again a consequence of Theorem 4 in [3]. We will use the second order Hamiltonian

$$
H(y, p, X):=\min _{b \in B} \max _{a \in A}\left\{-\frac{1}{2} \operatorname{trace}\left(\sigma \sigma^{T}(y, a, b) X\right)-f(y, a, b) \cdot p-l(y, a, b)\right\}
$$

for $y, p \in \mathbb{R}^{m}$ and $X$ any symmetric $m \times m$ matrix.

Theorem 4 The following statements are equivalent.

(i) The lower game is ergodic, i.e., $v(T, x) \rightarrow$ const uniformly in $x$ as $T \rightarrow+\infty$.

(ii) $l-\operatorname{val} E\left[\delta \int_{0}^{\infty} l\left(y_{x}(t), a(t), b(t)\right) e^{-\delta t} d t\right] \rightarrow$ const uniformly in $x$ as $\delta \rightarrow 0+$.

(iii) The cell problem

$$
\lambda+H\left(y, \nabla \chi, D^{2} \chi\right)=0 \quad \text { in } \mathbb{R}^{m}, \quad \chi \mathbb{Z}^{m} \text {-periodic }
$$

has the property that

$\sup \{\lambda \mid$ there is a viscosity subsolution of $(4.2)\}$

$$
=\inf \{\lambda \mid \text { there is a viscosity supersolution of (4.2) }\} \text {. }
$$

If one of the above assertions is true, then the constants in (i) and (ii) are equal and they coincide with the number defined by (4.3).

Proof The proof is the same as that of Theorem 2 after recalling that $w(t, y):=t v(t, y)$ is the viscosity solution of

$$
w_{t}+H\left(y, D_{y} w, D_{y y}^{2} w\right)=0 \quad \text { in }(0,+\infty) \times \mathbb{R}^{m}, \quad w(0, y)=0,
$$

and $w_{\delta}(x):=l-\operatorname{val} E\left[\int_{0}^{\infty} l\left(y_{x}(t), a(t), b(t)\right) e^{-\delta t} d t\right]$ is the viscosity solution of

$$
\delta w_{\delta}+H\left(y, D w_{\delta}, D^{2} w_{\delta}\right)=0 \quad \text { in } \mathbb{R}^{m},
$$

see [20].

Proposition 3 Assume the lower game is ergodic. Then $\lambda=\lim _{T \rightarrow \infty} v(T, x)$ satisfies

$$
\min _{x} \max _{b \in B} \min _{a \in A} l(x, a, b) \leq \lambda \leq \max _{x} \max _{b \in B} \min _{a \in A} l(x, a, b) .
$$

If, moreover,

$\min _{b \in B} \max _{a \in A}\left\{-\frac{1}{2} \operatorname{trace}\left(\sigma \sigma^{T}(y, a, b) X\right)-f(x, a, b) \cdot p-l(x, a, b)\right\} \geq \min _{b \in B} \max _{a \in A}\{-l(x, a, b)\} \quad \forall x, p, X$, (respectively, $\leq$ ), then

$$
\lambda=\min _{x} \max _{b \in B} \min _{a \in A} l(x, a, b),
$$

(respectively, $\lambda=\max _{x} \max _{b} \min _{a} l(x, a, b)$ ). 
PROOF The proof is the same as that of Proposition 1, after observing that

$$
-H(y, 0,0)=\max _{b \in B} \min _{a \in A} l(x, a, b) .
$$

It is well known that nondegenerate diffusion processes are ergodic (in the standard sense). The next result states the ergodicity of games involving a controlled system affected by a nondegenerate diffusion, with no controllability assumptions. It is a consequence of Theorem 7.1 in our paper [4], see also [3]. Earlier related results are due to Evans [16] and Arisawa and Lions [].

Theorem 5 Assume that, for some $v>0$, the minimal eigenvalue of the matrix $\sigma \sigma^{T}(y, a, b)$ is larger than $v$, for all $y \in \mathbb{R}^{m}, a \in A, b \in B$. Then the lower game is ergodic.

PROOF By means of regularity estimates for viscosity solutions of fully nonlinear uniformly elliptic PDEs, Theorem 7.1 in [ $\$$ ] shows that there exists a (unique) constant $\lambda$ such that the cell problem (4.2) has a continuous viscosity solution $\chi$. Then $u(t, y):=\lambda t+\chi(y)$ solves

$$
u_{t}+H\left(y, D_{y} u, D_{y y}^{2} u\right)=0 \quad \text { in }(0,+\infty) \times \mathbb{R}^{m}, \quad u(0, y)=\chi(y) .
$$

Since $w(t, y):=t v(t, y)$ solves (4.4), by the comparison principle for viscosity solutions of Cauchy problems we get

$$
u(t, y)-\max \chi \leq t v(t, y) \leq u(t, y)+\min \chi \quad \forall t, y .
$$

Therefore $v(t, \cdot) \rightarrow \lambda$ uniformly as $t \rightarrow \infty$.

The next result is an extension to stochastic games of Theorem 3 . We say that the system (4.1) is bounded-time controllable by the first player if for some $S>0$ and for all $x, \widetilde{x} \in \mathbb{R}^{m}$ there is a strategy $\widetilde{\alpha} \in \Gamma$ such that for all admissible control functions $b \in \mathcal{B}$

$$
\exists t^{\#}=t^{\#}(x, \widetilde{x}, \widetilde{\alpha}, b) \leq S \text { such that } y_{x}\left(t^{\#}\right)-\widetilde{x} \in \mathbb{Z}^{m} \text { almost surely, }
$$

where $y_{x}(\cdot)$ is the solution of (4.1) corresponding to the controls $\widetilde{\alpha}[b]$ and $b$.

Theorem 6 If the system (4.1) is bounded-time controllable by the first player, then the lower game is ergodic.

PROOF We follow the argument of the proof of Theorem 1. We use the characterization (ii) of ergodicity in Theorem $\sharp$, and we call $w_{\delta}$ the lower value function of the discounted infi nite horizon problem, namely,

$$
w_{\delta}(x):=\inf _{\alpha \in \Gamma} \sup _{b \in \mathcal{B}} E\left[\int_{0}^{\infty} l\left(y_{x}(t), \alpha[b](t), b(t)\right) e^{-\delta t} d t\right] .
$$

The main tool of the proof is the following Dynamic Programming Principle for stochastic games due to Swiech, Corollary 2.6 (iii) of [36],

$$
w_{\delta}(x)=\inf _{\alpha \in \Gamma} \sup _{b \in \mathcal{B}} \inf _{0 \leq t<\infty} E\left\{\int_{0}^{t} l\left(y_{x}(s), \alpha[b](s), b(s)\right) e^{-\delta s} d s+e^{-\delta t} w_{\delta}\left(y_{x}(t)\right)\right\} .
$$


For fi xed $x, \widetilde{x}$ we take a strategy $\widetilde{\alpha} \in \Gamma$ such that (4.7) holds. Then (4.8) and the periodicity of $w_{\delta}$ give

$$
w_{\delta}(x) \leq \sup _{b \in \mathcal{B}} E\left\{\int_{0}^{t^{\#}} l\left(y_{x}(s), \widetilde{\alpha}[b](s), b(s)\right) e^{-\delta s} d s+e^{-\delta t^{\#}} w_{\delta}(\widetilde{x})\right\},
$$

where $y_{x}(\cdot)$ is the trajectory of $(4.1)$ with the controls $\widetilde{\alpha}[b]$ and $b$. Now the proof of Theorem 3 shows that, along a sequence $\delta_{k} \rightarrow 0, \delta_{k} w_{\delta_{k}} \rightarrow \mu$ uniformly.

Finally, the proof that $\mu$ dos not depend on the sequence $\delta_{k}$ is also the same as in Theorem 8, with the new cell problem (4.2) and using the equation (4.5) satisfi ed by $w_{\delta}$.

The last result of the section is a stochastic counterpart of Proposition 2. We take a controlled diffusion of the form

$$
\begin{cases}d y^{A}(t)=f_{A}(y(t), a(t), b(t)) d t+\sigma_{A}(y(t), a(t), b(t)) d W_{A}(t), & y^{A}(0)=x^{A} \in \mathbb{R}^{m_{A}} \\ d y^{B}(t)=f_{B}(y(t), a(t), b(t)) d t+\sigma_{B}(y(t), a(t), b(t)) d W_{B}(t), & y^{B}(0)=x^{B} \in \mathbb{R}^{m_{B}} \\ y(t)=\left(y^{A}(t), y^{B}(t)\right) & \end{cases}
$$

and we assume that the state variables $y^{A}$ are asymptotically controllable by the fi rst player, and the variables $y^{B}$ are asymptotically controllable by the second, in the following sense. There exists a function $\eta:[0, \infty) \rightarrow[0, \infty)$ with $\lim _{T \rightarrow \infty} \eta(T)=0$, and for all $x^{A}, \widetilde{x}^{A} \in \mathbb{R}^{m_{A}}, x^{B} \in \mathbb{R}^{m_{B}}$, there is a strategy $\widetilde{\alpha} \in \Gamma$, such that, for $x=\left(x^{A}, x^{B}\right)$,

$$
E\left[\frac{1}{T} \int_{0}^{T} \min _{k^{A} \in \mathbb{Z}^{m_{A}}}\left|y_{x}^{A}(t)-\widetilde{x}^{A}-k^{A}\right| d t\right] \leq \eta(T), \forall b \in \mathcal{B},
$$

whereas for all $x^{B}, \widetilde{x}^{B} \in \mathbb{R}^{m_{B}}, x^{A} \in \mathbb{R}^{m_{A}}$, there is a strategy $\widetilde{\beta} \in \Delta$ such that

$$
E\left[\frac{1}{T} \int_{0}^{T} \min _{k^{B} \in \mathbb{Z}^{m_{B}}}\left|y_{x}^{B}(t)-\widetilde{x}^{B}-k^{B}\right| d t\right] \leq \eta(T), \forall a \in \mathcal{A} .
$$

As in Section 2 we assume that the running cost does not depend on the controls and has a saddle point. The Isaacs condition now is

$$
\begin{gathered}
\min _{b \in B} \max _{a \in A}\left\{-\frac{1}{2} \operatorname{trace}\left(\sigma \sigma^{T}(y, a, b) X\right)-f(y, a, b) \cdot p-l(y, a, b)\right\}= \\
\max _{a \in A} \min _{b \in B}\left\{-\frac{1}{2} \operatorname{trace}\left(\sigma \sigma^{T}(y, a, b) X\right)-f(y, a, b) \cdot p-l(y, a, b)\right\} .
\end{gathered}
$$

Proposition 4 Assume the system (4.1) is of the form (4.9) with $y^{A}$ and $y^{B}$ asymptotically controllable, respectively, by the first and by the second player. Suppose also $l=l\left(y^{A}, y^{B}\right)$ satisfies (2.17) and (4.11) holds. Then the lower game is ergodic and its value converges to the value of the static game with payoff $l$, that is,

$$
\lim _{T \rightarrow \infty} v\left(T, x^{A}, x^{B}\right)=\bar{l}, \quad \text { uniformly in }\left(x^{A}, x^{B}\right) \in \mathbb{R}^{m} .
$$

REMARK If the system governing $y^{A}$ is bounded-time controllable by the first player and also stoppable, i.e.,

$$
\forall x \in \mathbb{R}^{m}, \forall b \in B, \exists a \in A: f_{A}(x, a, b)=0, \sigma_{A}(x, a, b)=0,
$$


then the variables $y^{A}$ are asymptotically controllable, because $\widetilde{x}^{A}$ can be reached from $x^{A}$ in a time smaller than $S$ and then the first player can keep $y^{A}(t)=\widetilde{x}^{A}$ for all later times $t$. As in the deterministic case, an optimal strategy for each player is a turnpike: driving the system to a saddle point and stopping there.

REMARK The proof of Proposition $\emptyset$ shows also that the upper game is ergodic and the upper value $u-\operatorname{val} J(T, x)$ converge uniformly to the saddle $\bar{l}$ as $T \rightarrow \infty$.

\section{Acknowledgments}

We thank Wendell Fleming and Bill McEneaney for some remarks on their paper [19], and Pierre Cardaliaguet for useful comments on a preliminary version of this paper. Olivier Alvarez was partially supported by the research project ACI-JC 1025 "Dislocation dynamics" of the French Ministry of Education. Martino Bardi was partially supported by the research project "Viscosity, metric, and control theoretic methods for nonlinear partial differential equations" of the italian M.I.U.R.

\section{References}

[1] O. Alvarez, Homogenization of Hamilton-Jacobi equations in perforated sets, J. Differential Equations 159 (1999), 543-577.

[2] O. Alvarez and M. Bardi, Viscosity solutions methods for singular perturbations in deterministic and stochastic control, SIAM J. Control Optim. 40 (2001), 1159-1188.

[3] O. Alvarez and M. Bardi, Singular perturbations of degenerate parabolic PDEs: a general convergence result, Arch. Rational Mech. Anal. 170 (2003), 17-61.

[4] O. Alvarez and M. Bardi, Ergodicity, stabilization, and singular perturbations of Bellman-Isaacs equations, Tech. report, University of Padova, 2004, to appear.

[5] O. Alvarez and M. Bardi, Ergodic problems in differential games, Tech. report, University of Padova, 2004, to appear.

[6] O. Alvarez, M. Bardi, and C. Marchi, Multiscale problems and homogenization for second-order Hamilton-Jacobi equations, Tech. report, University of Padova, 2004, to appear.

[7] M. Arisawa, Ergodic problem for the Hamilton-Jacobi-Bellman equation II, Ann. Inst. H. Poincaré Anal. Non Linéaire 15 (1998), 1-24.

[8] M. Arisawa and P.-L. Lions, On ergodic stochastic control, Comm. Partial Differential Equations 23 (1998), 2187-2217.

[9] Z. Artstein and V. Gaitsgory, The value function of singularly perturbed control systems, Appl. Math. Optim. 41 (2000), 425-445.

[10] M. Bardi and I. Capuzzo-Dolcetta, Optimal control and viscosity solutions of Hamilton-Jacobi-Bellman equations, Birkhäuser, Boston, 1997.

[11] A. Bensoussan, Perturbation methods in optimal control, Wiley/Gauthiers-Villars, Chichester, 1988.

[12] R. Buckdahn and N. Ichihara, Limit theorems for controlled backward sdes and homogenization of hamilton-jacobi-bellman equations, Tech. report, 2004. 
[13] D.A. Carlson and A.B. Haurie, A turnpike theory for infi nite horizon open-loop differential games with decoupled controls, New trends in dynamic games and applications (G.J. Olsder, ed.), Ann. Internat. Soc. Dynam. Games, no. 3, Birkhäuser Boston, Boston, MA, 1995, pp. 353-376.

[14] D.A. Carlson, A.B. Haurie, and A. Leizarowitz, Infi nite horizon optimal control: Deterministic and stochastic systems, Springer-Verlag, Berlin, 1991.

[15] F. Colonius and W. Kliemann, The dynamics of control, Birkhäuser, Boston, 2000.

[16] L. Evans, The perturbed test function method for viscosity solutions of nonlinear P.D.E., Proc. Roy. Soc. Edinburgh Sect. A 111 (1989), 359-375.

[17] L. Evans, Periodic homogenisation of certain fully nonlinear partial differential equations, Proc. Roy. Soc. Edinburgh Sect. A 120 (1992), 245-265.

[18] L. Evans and P. E. Souganidis, Differential games and representation formulas for solutions of Hamilton-Jacobi-Isaacs equations, Indiana Univ. Math. J. 33 (1984), 773-797.

[19] W. H. Fleming and W.M. McEneaney, Risk-sensitive control on an infi nite time horizon, SIAM J. Control Optim. 33 (1995), 1881-1915.

[20] W. H. Fleming and P. E. Souganidis, On the existence of value functions of two-players, zero-sum stochastic differential games, Indiana Univ. Math. J. 38 (1989), 293-314.

[21] V. Gaitsgory, Limit Hamilton-Jacobi-Isaacs equations for singularly perturbed zero-sum differential games, J. Math. Anal. Appl. 202 (1996), 862-899.

[22] L. Grüne, On the relation between discounted and average optimal value functions, J. Differential Equations 148 (1998), 65-99.

[23] H. Ishii, Homogenization of the Cauchy problem for Hamilton-Jacobi equations, Stochastic analysis, control, optimization and applications. A volume in honor of Wendell H. Fleming (W.M. McEneaney, G. Yin, and Q. Zhang, eds.), Birkhäuser, Boston, 1999, pp. 305-324.

[24] Y. Kabanov and S. Pergamenshchikov, Optimal control of singularly perturbed linear stochastic systems, Stochastics Stochastics Rep. 36 (1991), 109-135.

[25] P.V. Kokotović, H.K. Khalil, and J. O'Reilly, Singular perturbation methods in control: analysis and design, Academic Press, London, 1986.

[26] H.J. Kushner, Weak convergence methods and singularly perturbed stochastic control and fi ltering problems, Birkhäuser, Boston, 1990.

[27] H.J. Kushner, Numerical approximations for stochastic differential games: the ergodic case, SIAM J. Control Optim. 42 (2004), 1911-1933.

[28] P.-L. Lions, G. Papanicolaou, and S. R. S. Varadhan, Homogeneization of Hamilton-Jacobi equations, Unpublished, 1986.

[29] C. Marchi, Homogenization for fully nonllinear parabolic equations, Nonlinear Anal. 60 (2005), 411-428.

[30] M. Quincampoix and F. Watbled, Averaging method for discontinuous mayer's problem of singularly perturbed control systems, Nonlinear Anal. 54 (2003), 819-837.

[31] P. Soravia, Pursuit-evasion problems and viscosity solutions of Isaacs equations, SIAM J. Control Optim. 31 (1993), 604-623.

[32] P. Soravia, Stability of dynamical systems with competitive controls: the degenerate case, J. Math. Anal. Appl. 191 (1995), 428-449. 
[33] S. Sorin, New approaches and recent advances in two-person zero-sum repeated games, Advances in dynamic games (A.S. Nowak and K. Szajowski, eds.), Ann. Internat. Soc. Dynam. Games, no. 7, Birkhäuser, Boston, 2005, pp. 67-93.

[34] N.N. Subbotina, Asymptotic properties of minimax solutions of Isaacs-Bellman equations in differential games with fast and slow motions, J. Appl. Math. Mech. 60 (1996), 883-890.

[35] N.N. Subbotina, Asymptotics for singularly perturbed differential games, Game theory and applications, Vol. VII, Nova Sci. Publ., Huntington, NY, 2001, pp. 175-196.

[36] A. Swiech, Another approach to the existence of value functions of stochastic differential games, J. Math. Anal. Appl. 204 (1996), 884-897. 